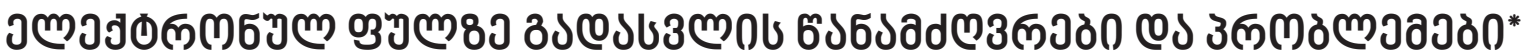

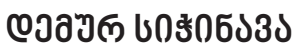

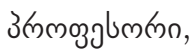

https://doi.org/10.35945/gb.2018.06.026

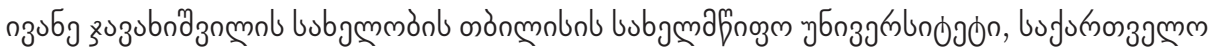

demur.sitchinava@tsu.ge

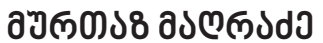

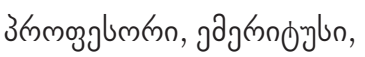

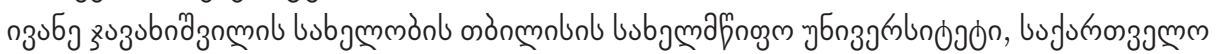

murtaz.maghradze@tsu.ge

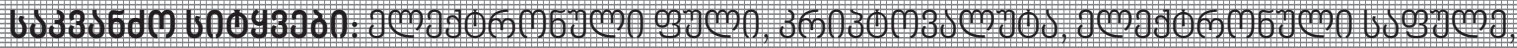

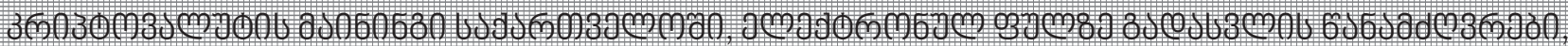

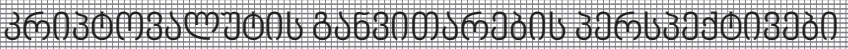

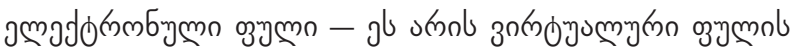

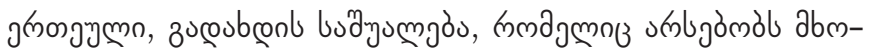

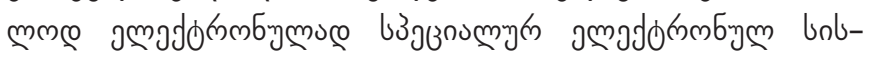

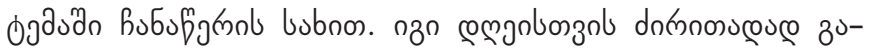
amngjб

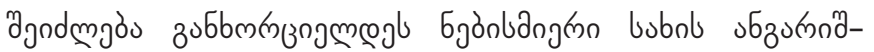

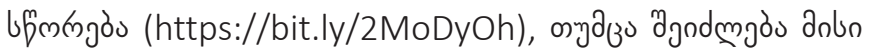

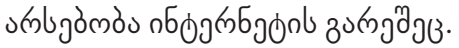

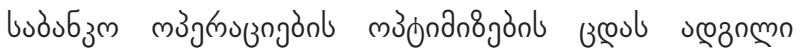

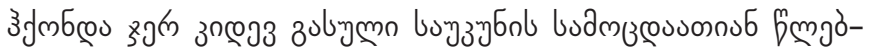

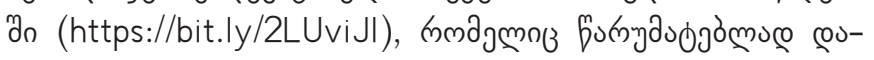

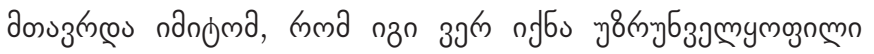

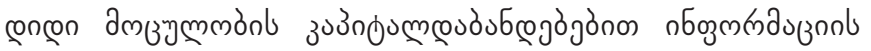

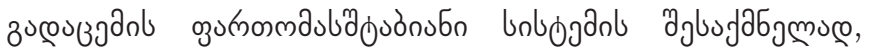

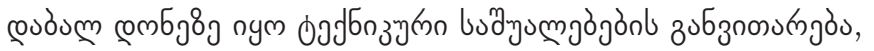

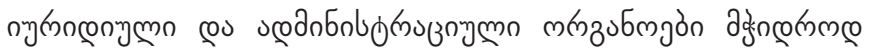

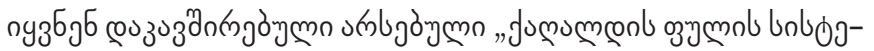

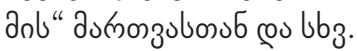

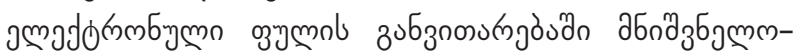

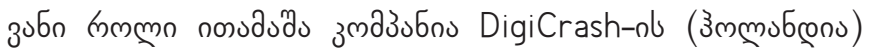

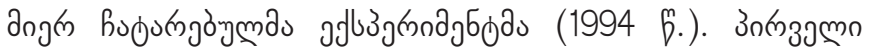

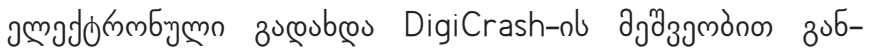

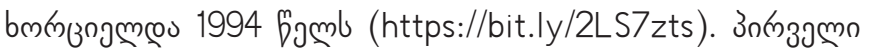

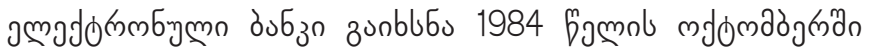

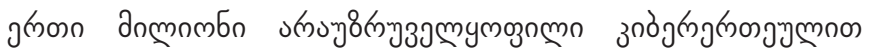

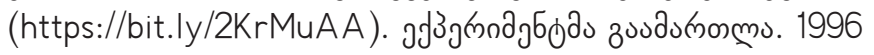

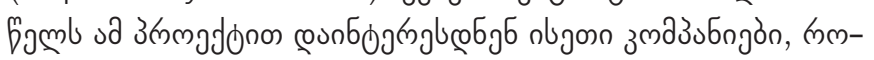

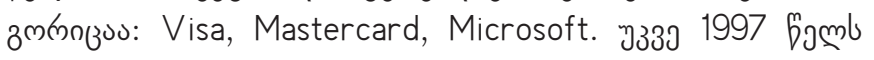

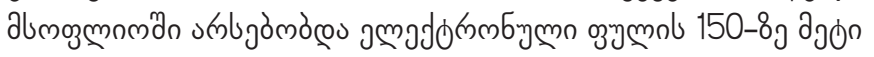
उஙnmgdon (https://bit.ly/2KrMuAA).

* ззмлззи д

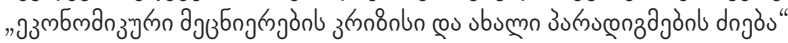

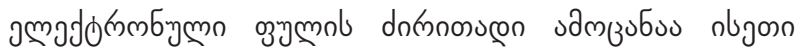

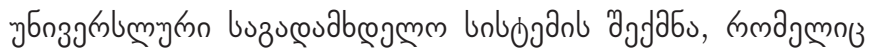

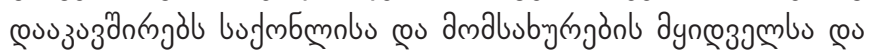

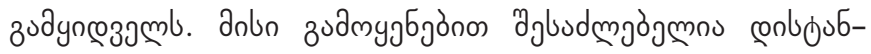

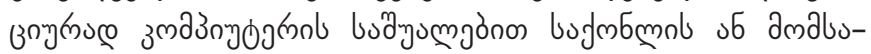

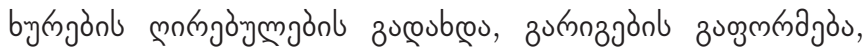

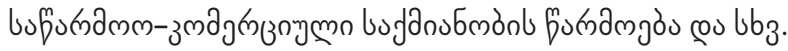

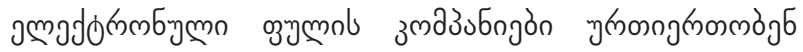

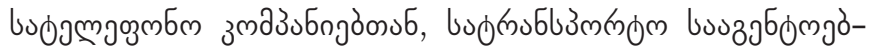

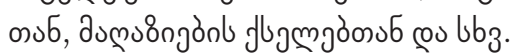

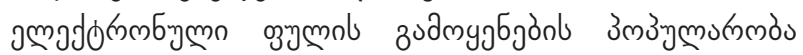
zubnbu8

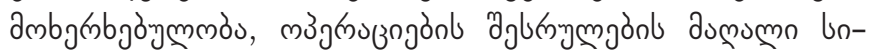

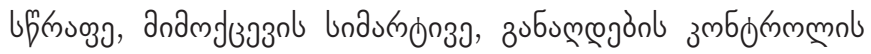

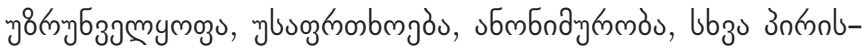

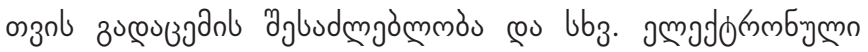

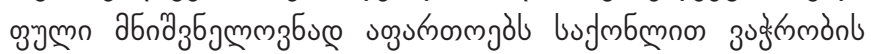

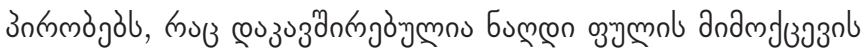

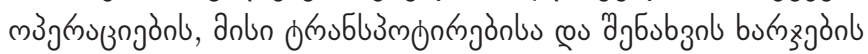

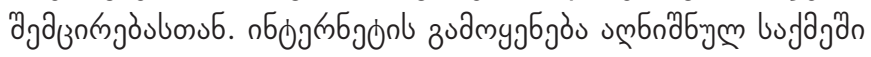

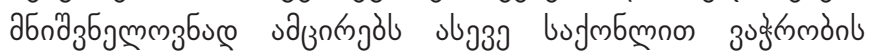
cosbuburnzgal.

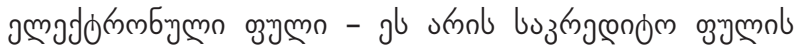

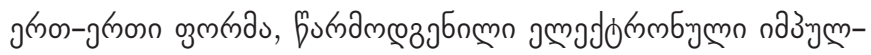

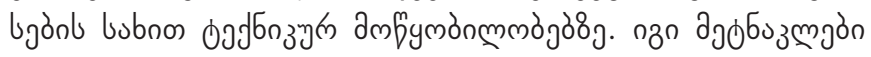

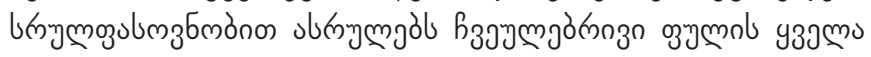

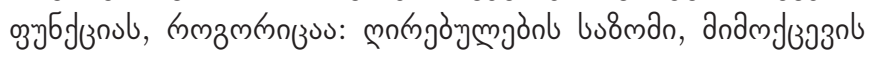

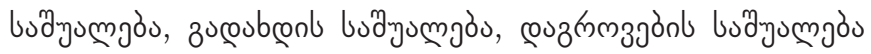

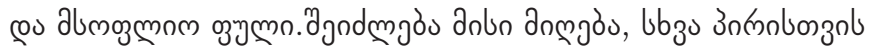

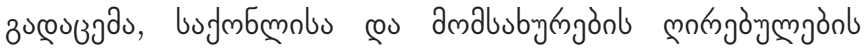

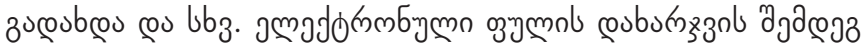

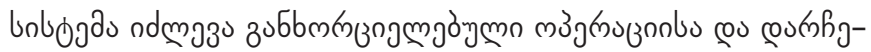

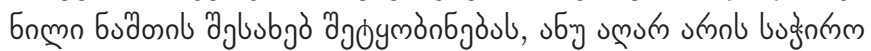




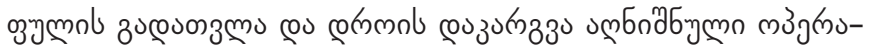

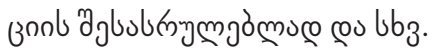

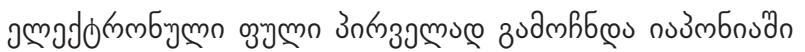

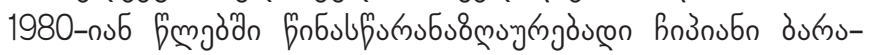

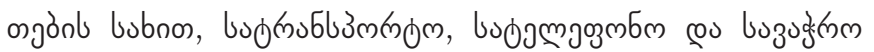

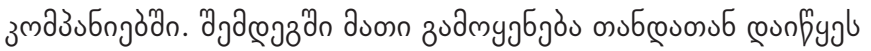

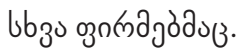

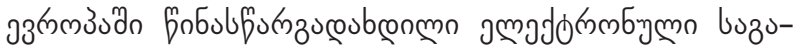

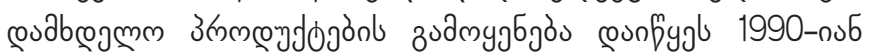

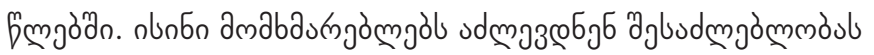

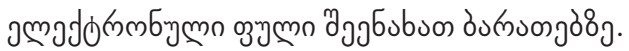

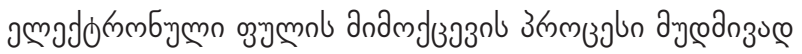

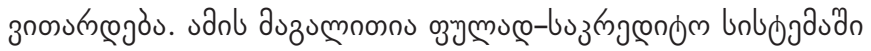
jмado

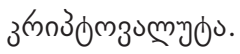

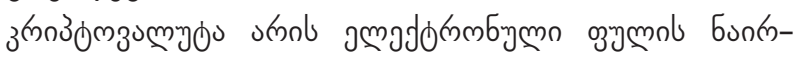

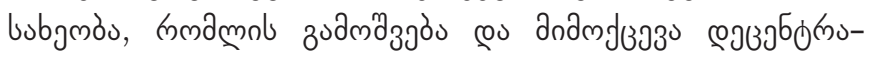

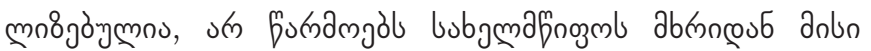

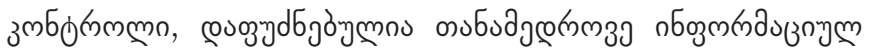

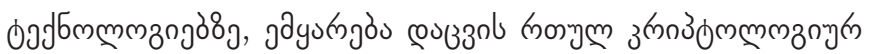

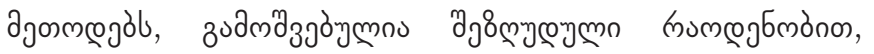

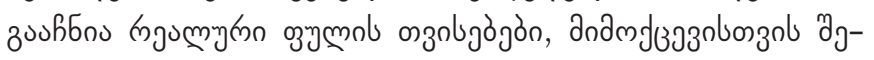

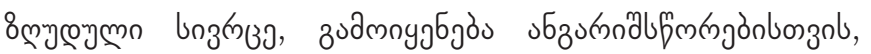

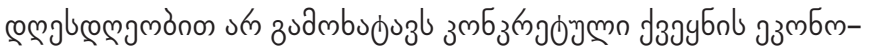

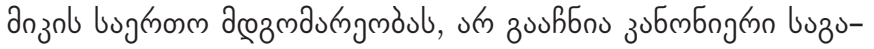

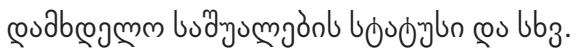

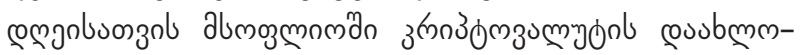

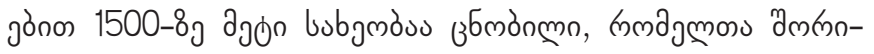

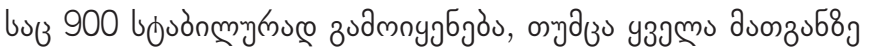

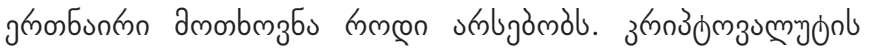

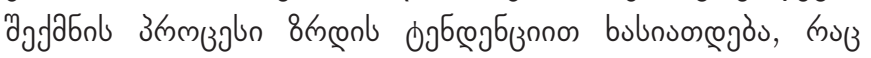

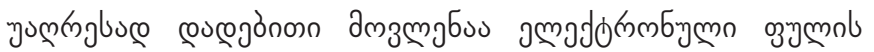

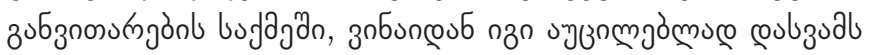

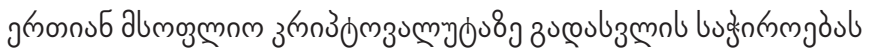
(https://bit.ly/2AIGLXt).

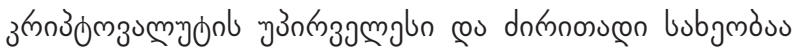

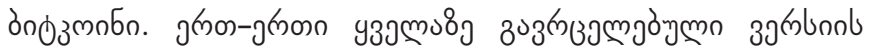

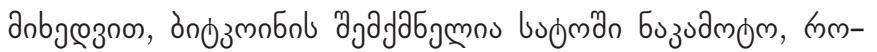

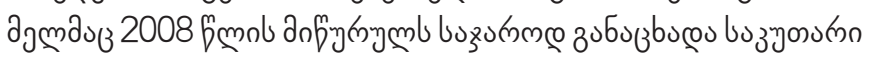
zudm zm

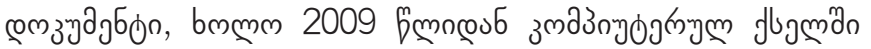

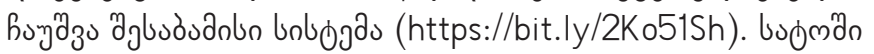

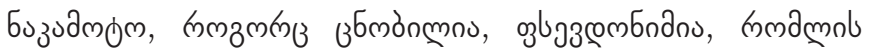

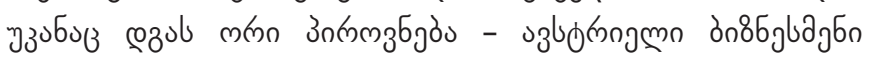

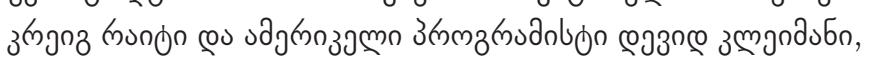

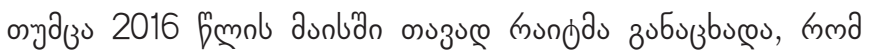

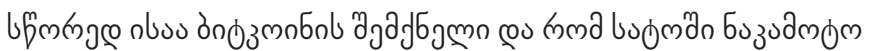

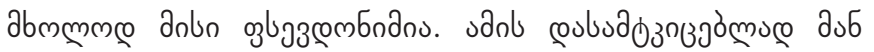

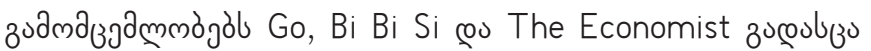

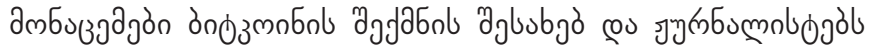

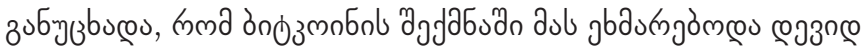
зmgुnaubn.

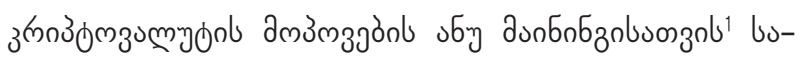

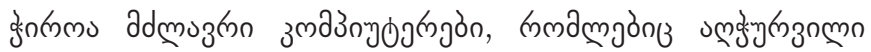

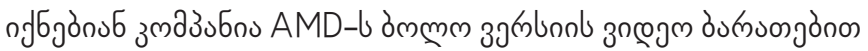

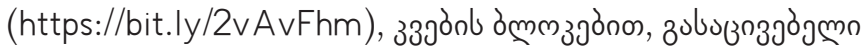

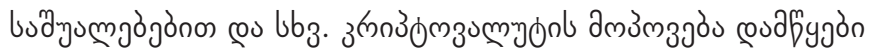

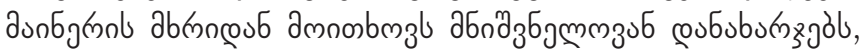

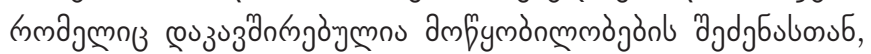

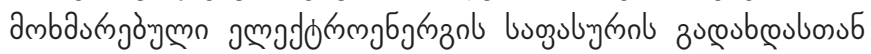
एos.. .

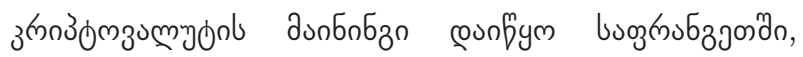
numubconuan eu bufurnoszamman 2014 famb buzyosumn

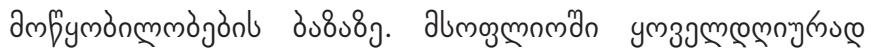

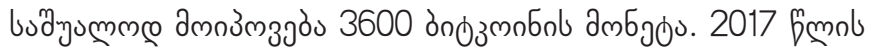

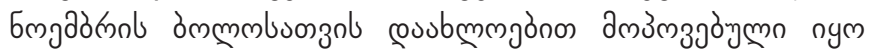

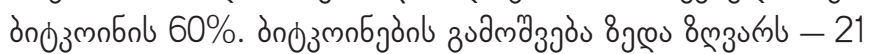

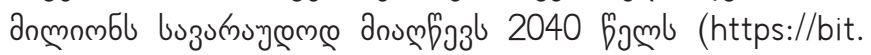
ly/2AL5omk).

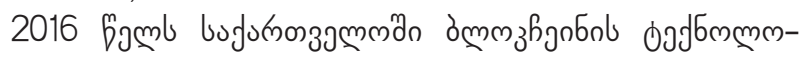

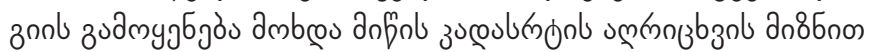

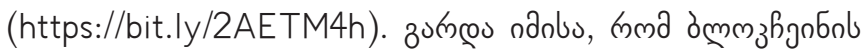

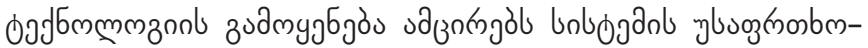

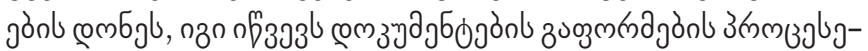

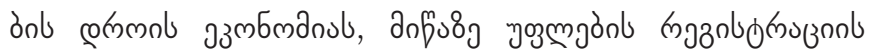

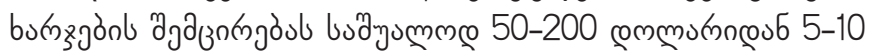

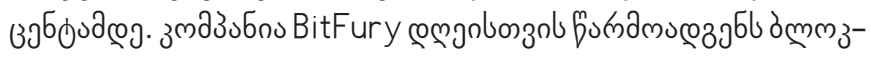

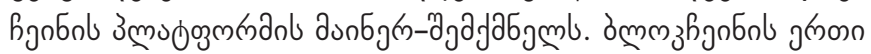

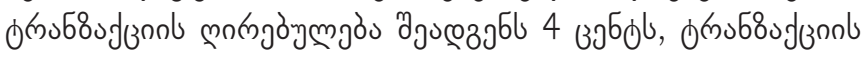
corm 3n-8 fyoll (https://bit.ly/2AETM4h).

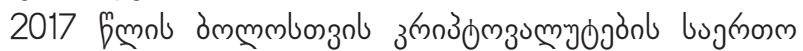

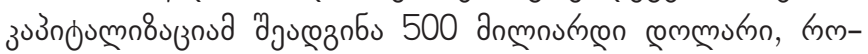

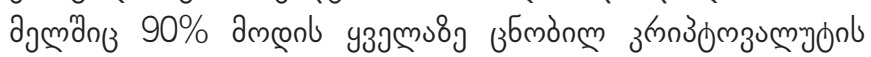
sogym 8 g: bitcoin - 57,5\%, Etereum - 12,8\%, bitcoin Cash 5.3\%, Ripple-3,8\%, IOTA -2,4\%, Dash-1,4\%, NEM-1,0\%, Monero - 1,0\%,NEO - 0,5\%, usbumhjon - 11,1\% (https://bit. ly/2LUvOr3).

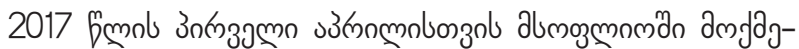

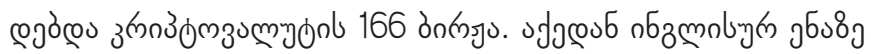

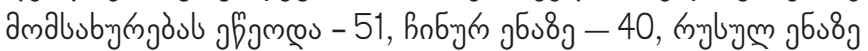

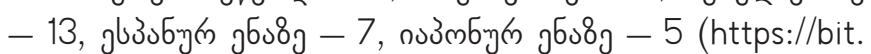
ly/2McdUfg).

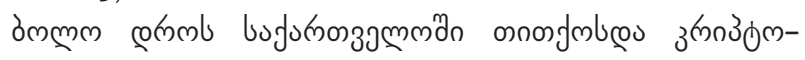

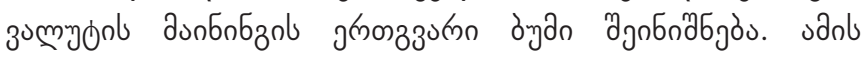

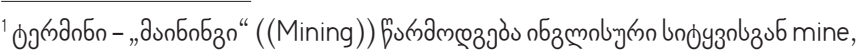

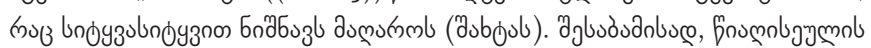

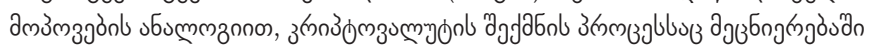

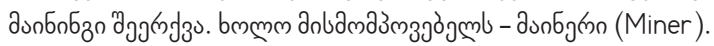




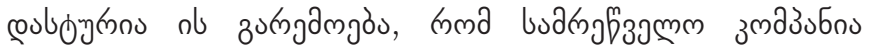

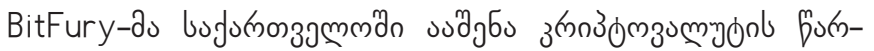

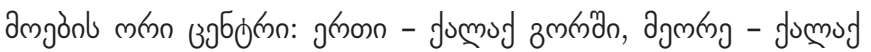

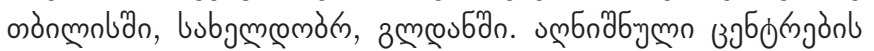

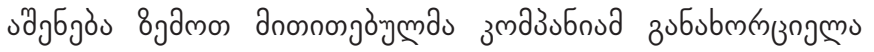

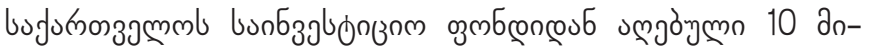

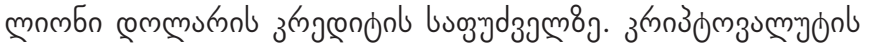

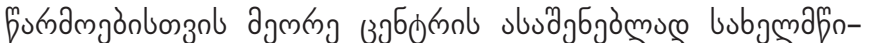

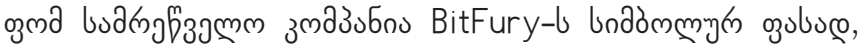

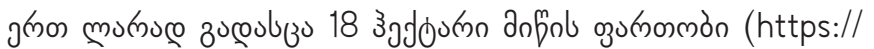

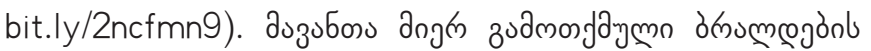

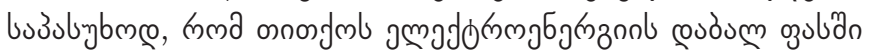

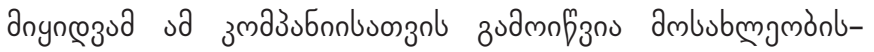
mzol jmadd

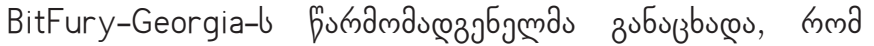

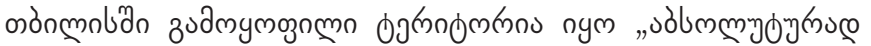

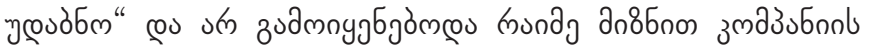

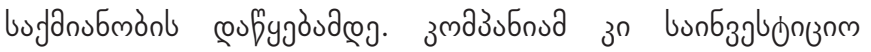

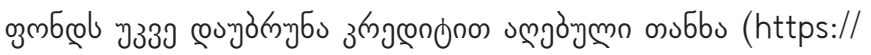

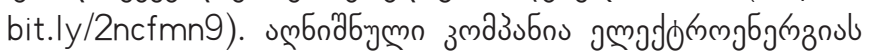

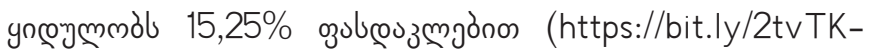

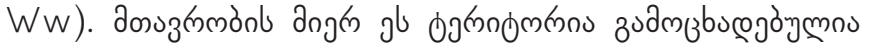

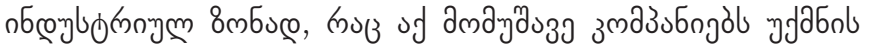

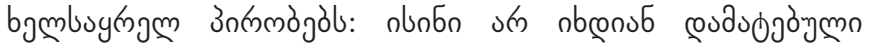

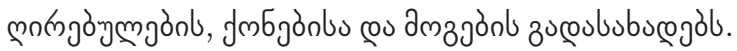

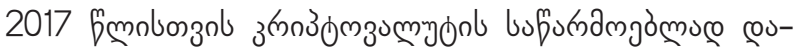

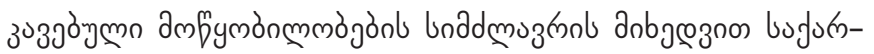

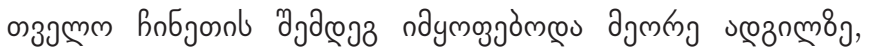

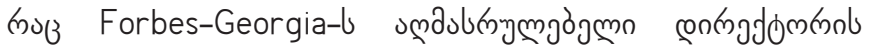

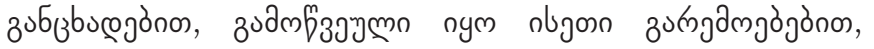

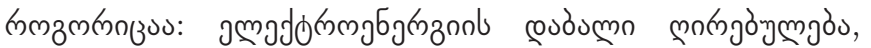

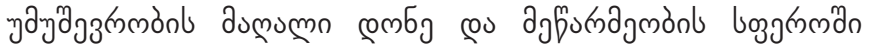

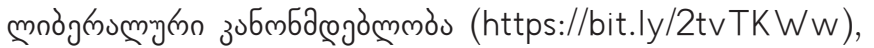

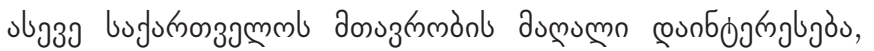

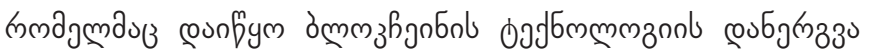

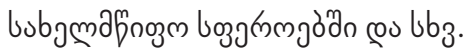

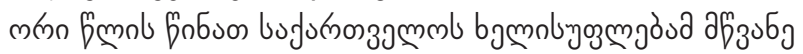

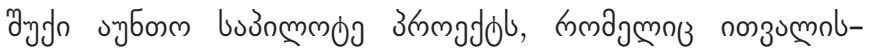

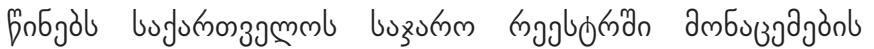

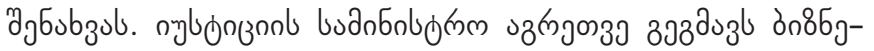

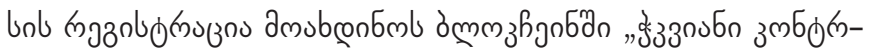

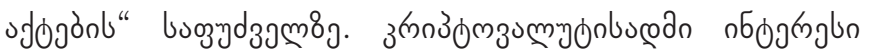

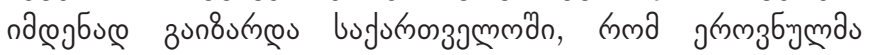

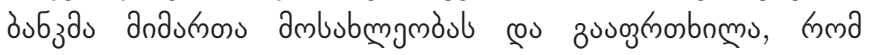

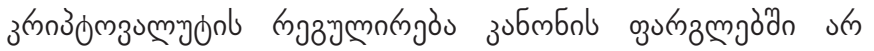

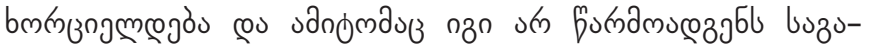

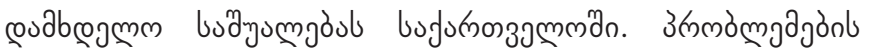

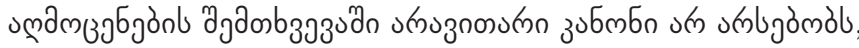

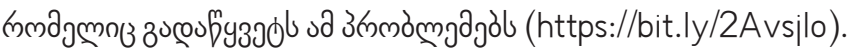

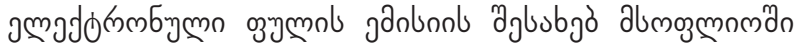

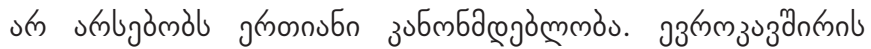

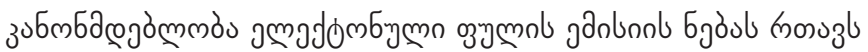

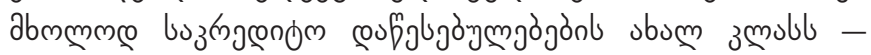

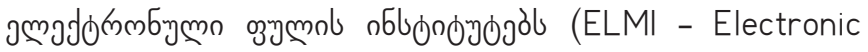

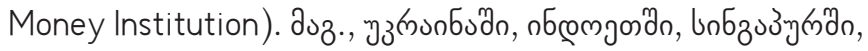

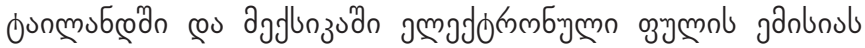

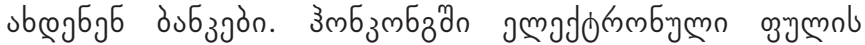

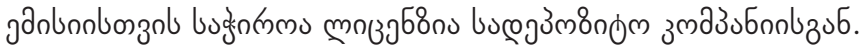

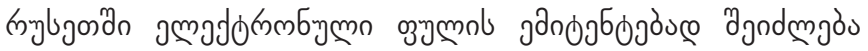
zuдmzn@

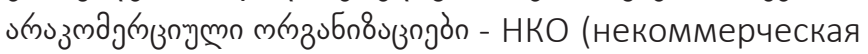

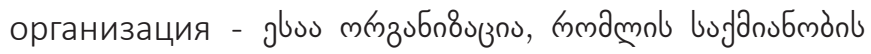

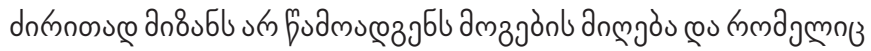

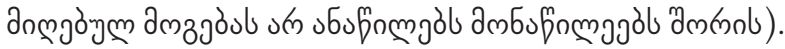

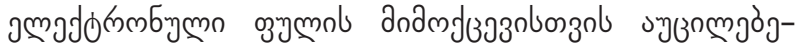

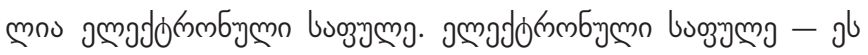

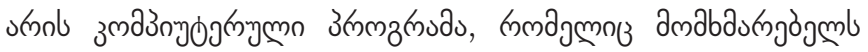

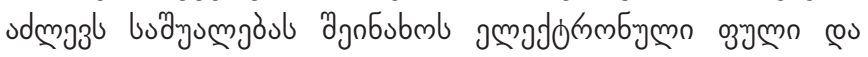

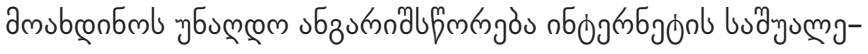

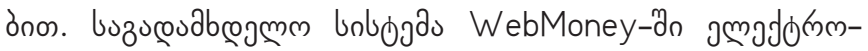

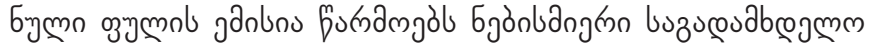

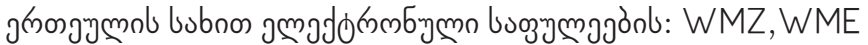

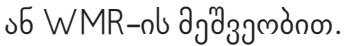

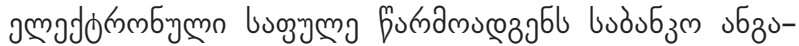

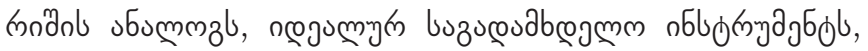

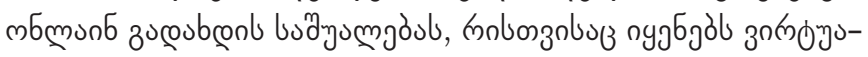

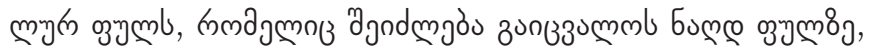

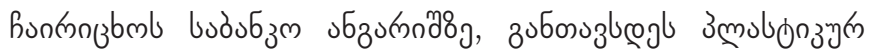
oumooson.

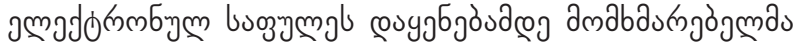

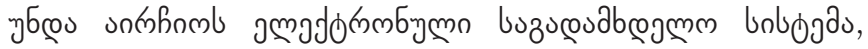

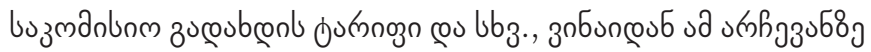

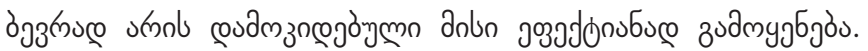

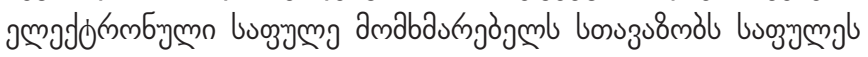

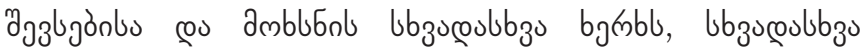

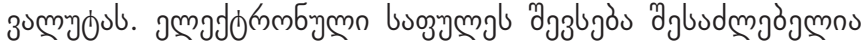

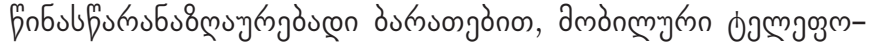

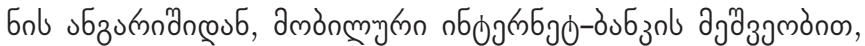

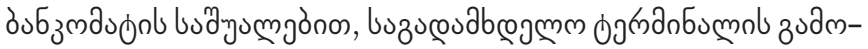

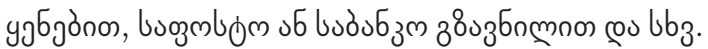

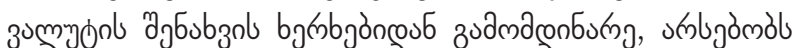

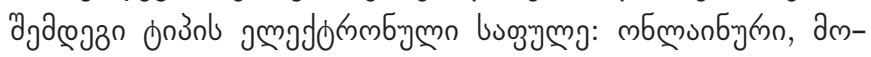

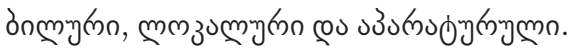

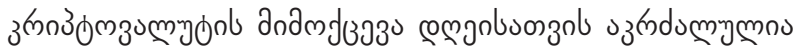

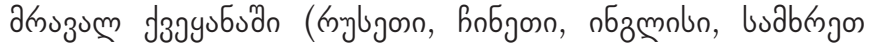

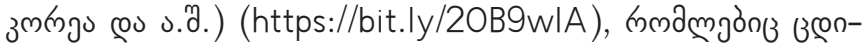

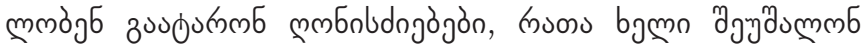

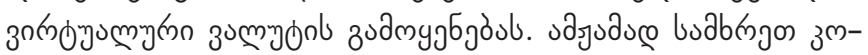




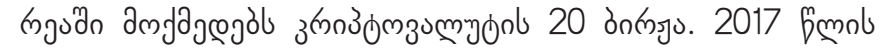

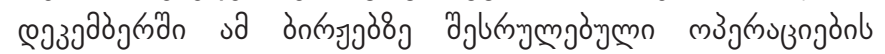

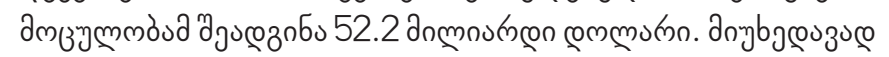

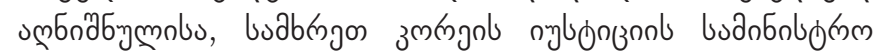

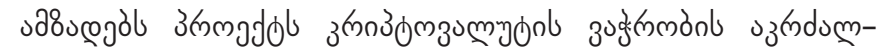

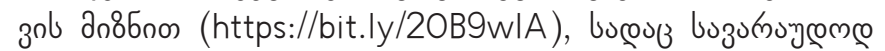

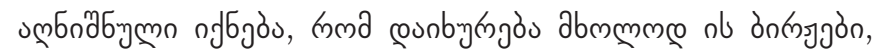

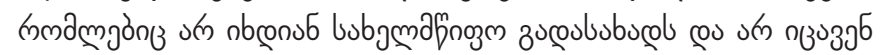

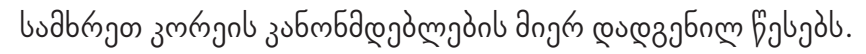

s ऊl

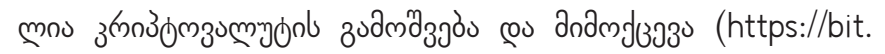

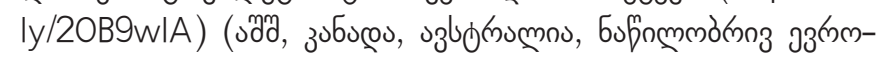

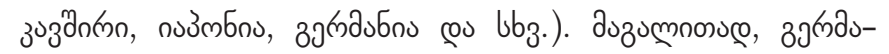

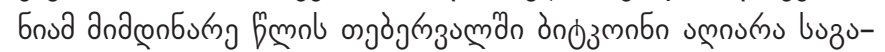

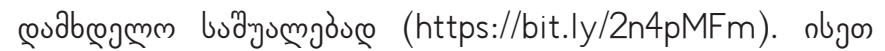

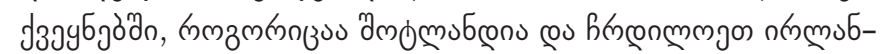

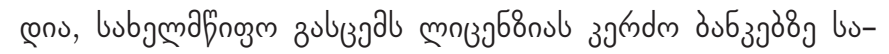

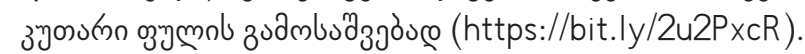

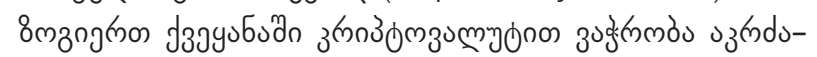

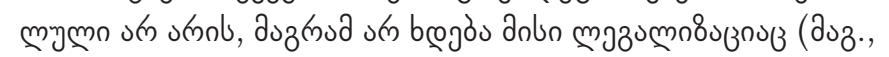

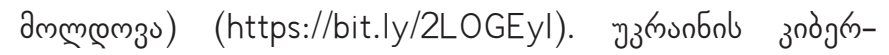

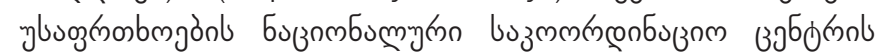

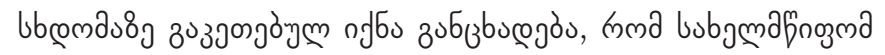

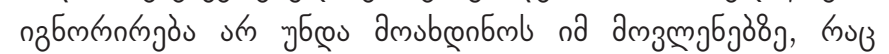

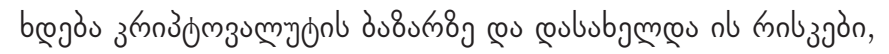

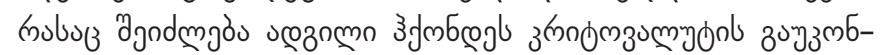

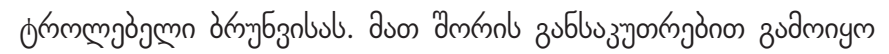

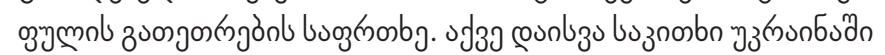

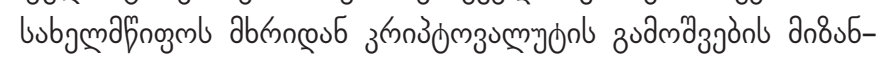

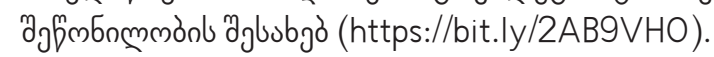

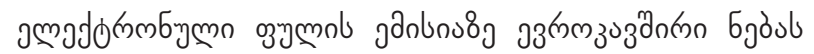

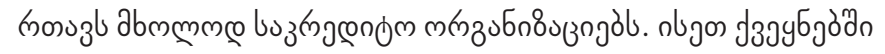

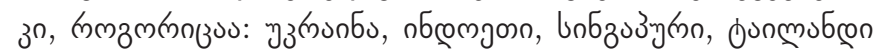

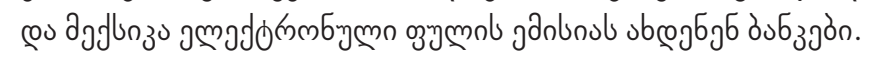

jmado

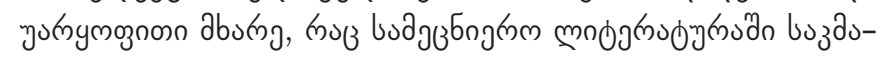

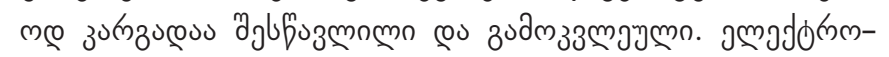

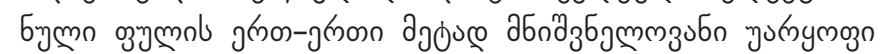

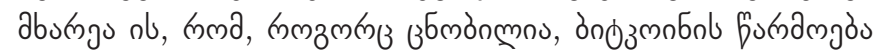

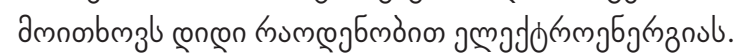

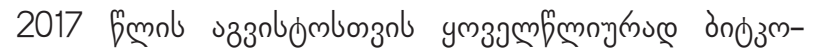

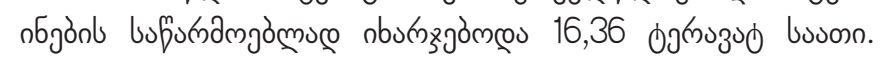

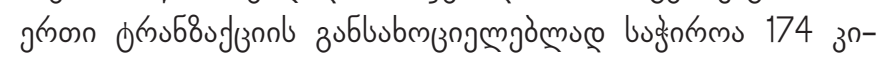

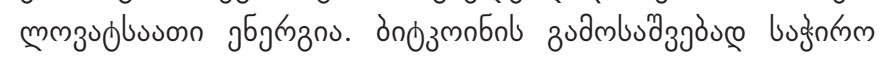

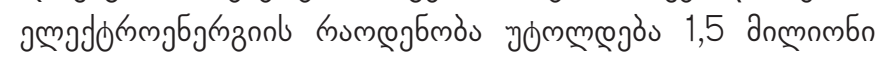

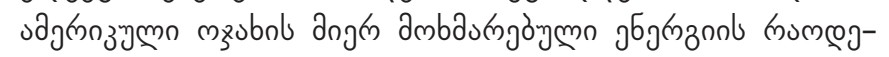

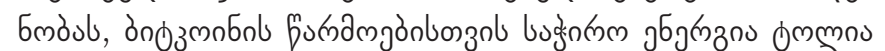

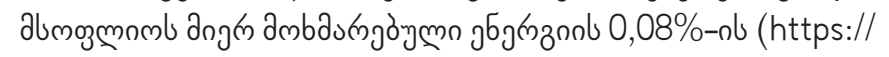
bit.ly/2ofPzOx).

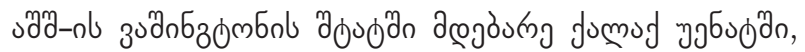
пмадgmbus a

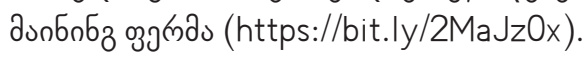

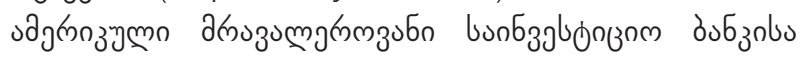

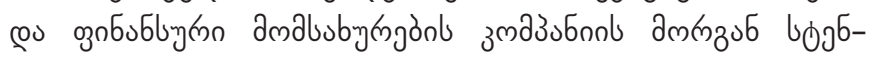

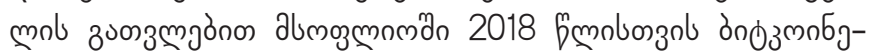

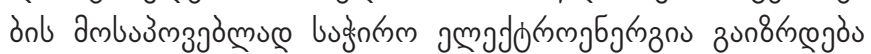

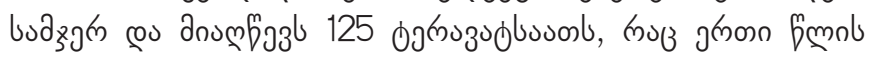

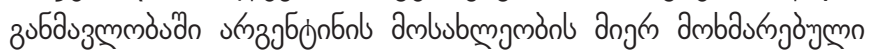

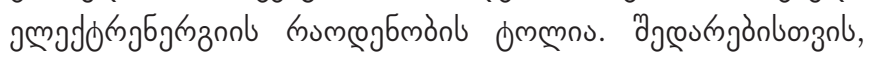

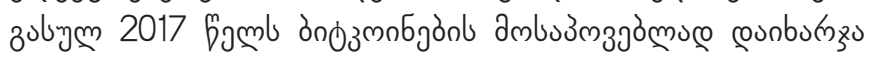

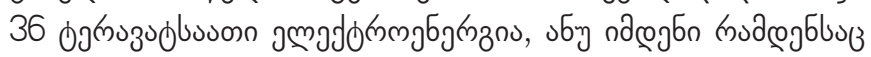

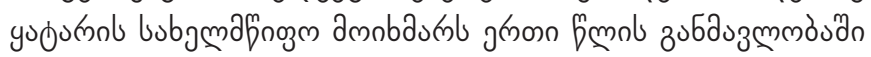
(https://bit.ly/2n4Hfxm).

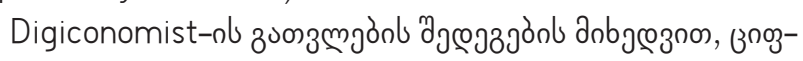

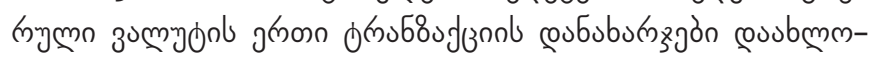

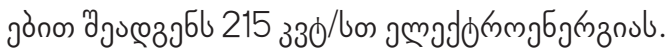

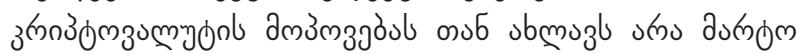

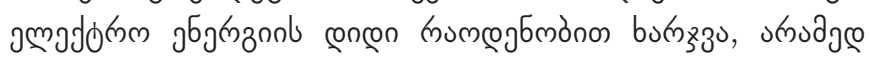

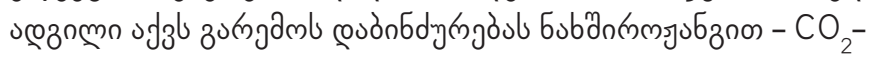

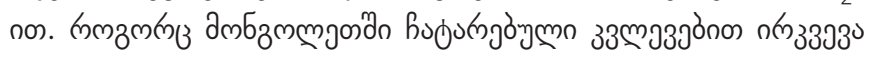

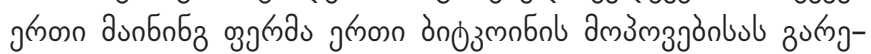

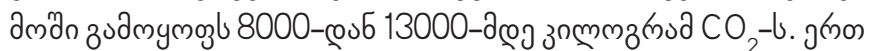
busoman z 24000-bit.ly/2LZr2HY).

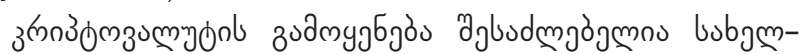

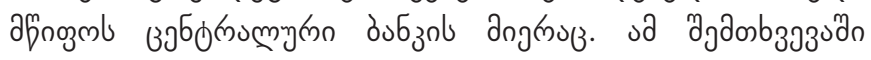

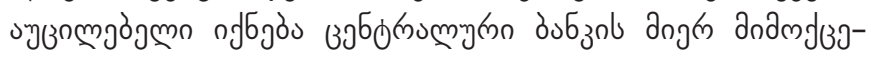

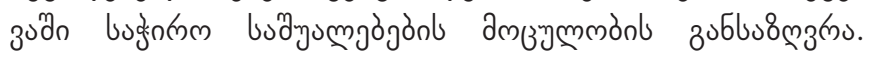

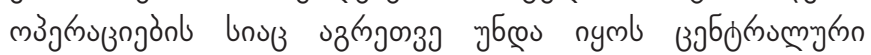

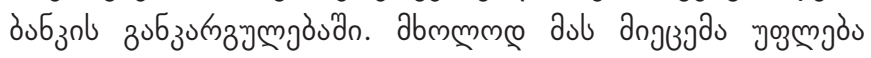

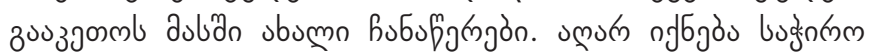

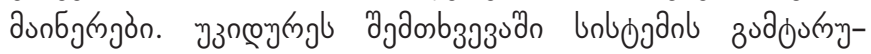

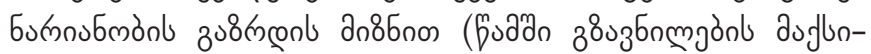

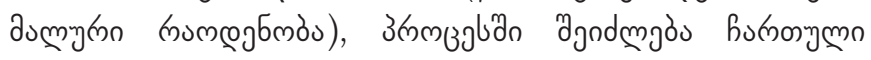

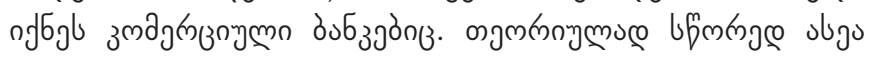

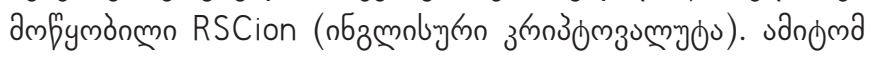

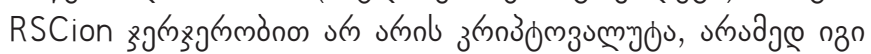

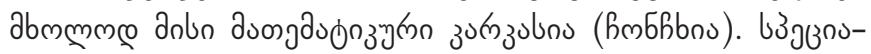

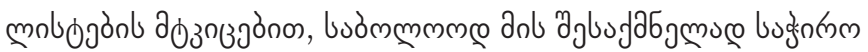

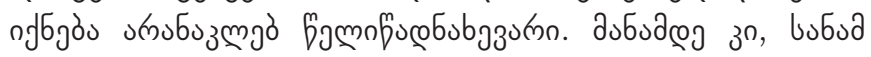

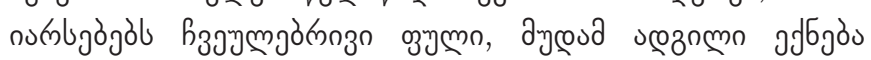

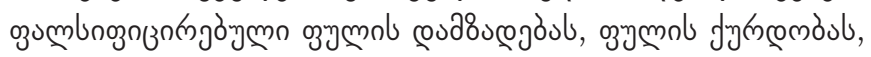

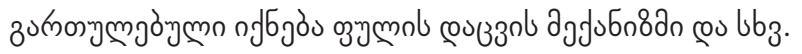

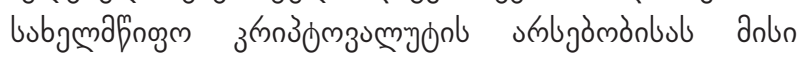

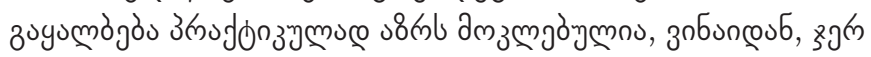

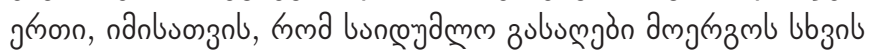

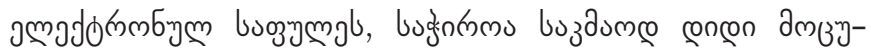

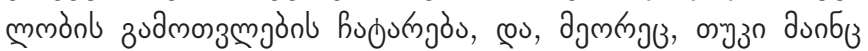




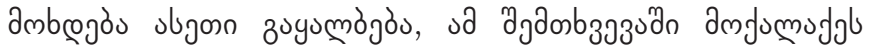

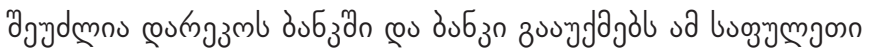

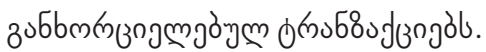

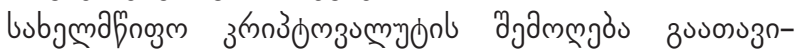

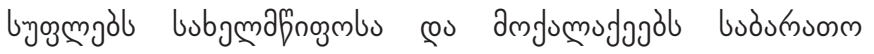

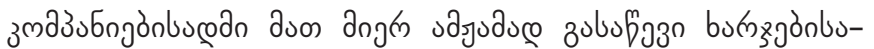

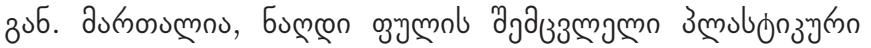

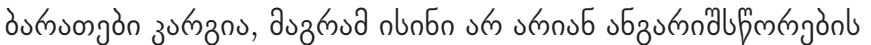

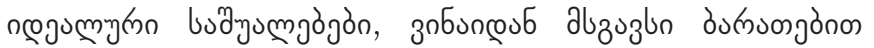

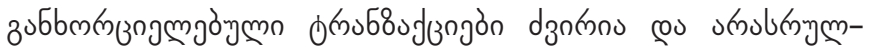
oुulusubnos.

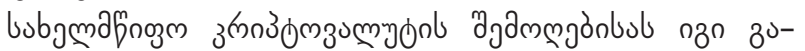

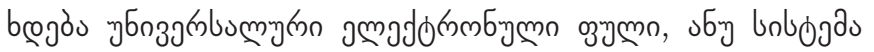

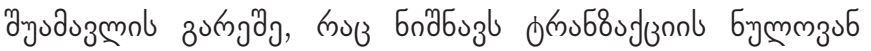

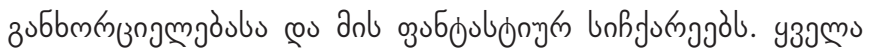

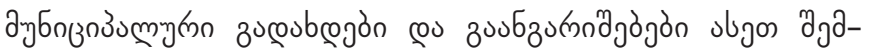

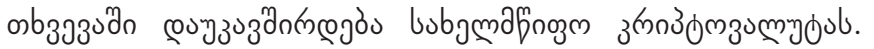

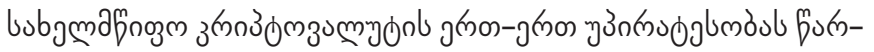

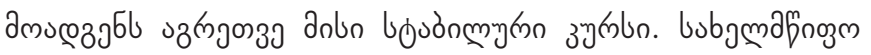

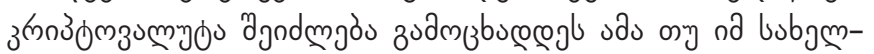

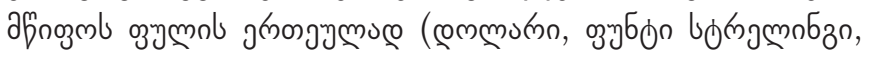

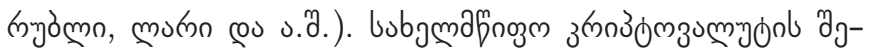

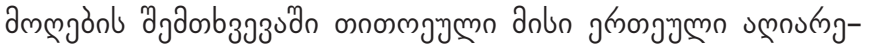

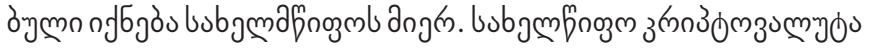

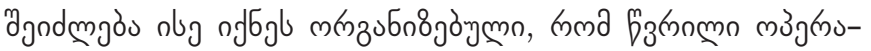

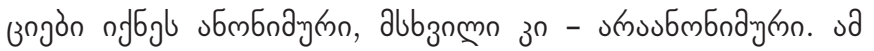

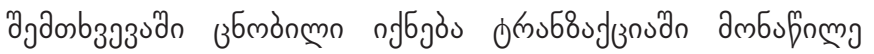

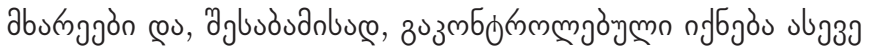

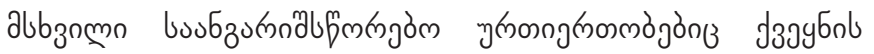

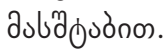

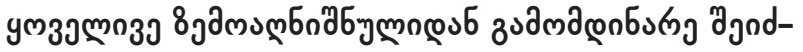

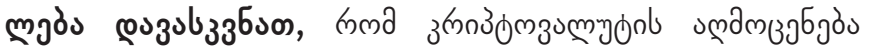

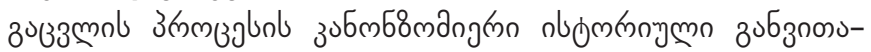

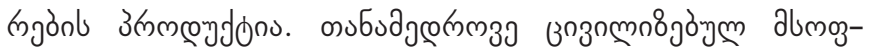

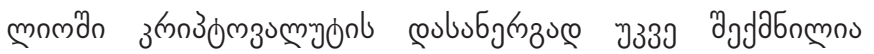

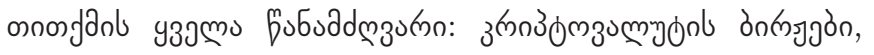

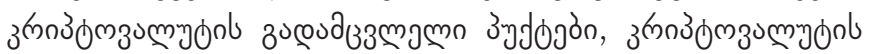

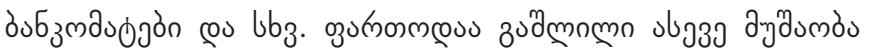

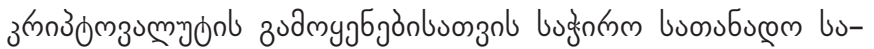
з јбмбдая

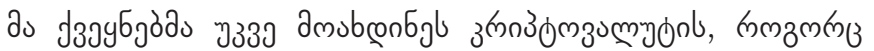

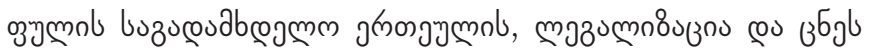

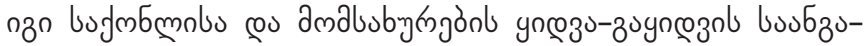

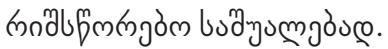

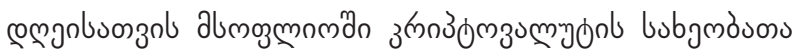

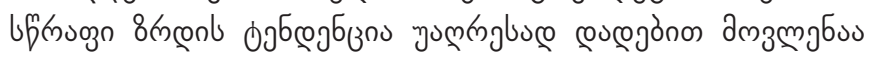
jmadd

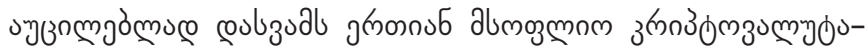

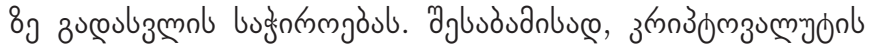

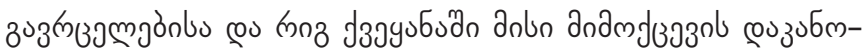

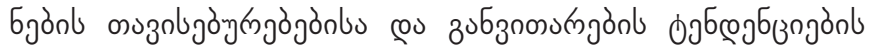

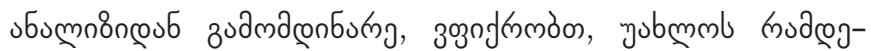

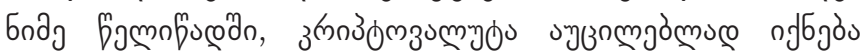

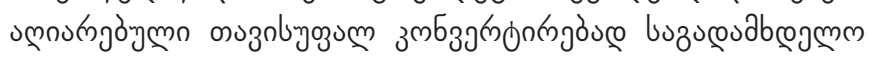

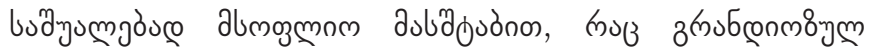

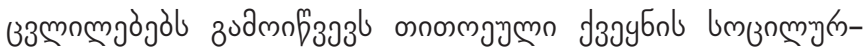

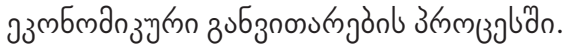

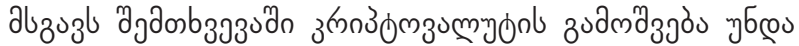

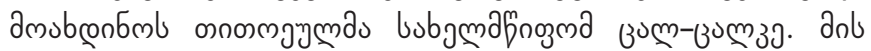

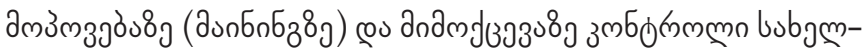

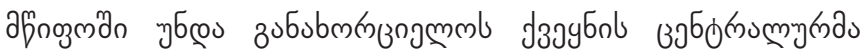

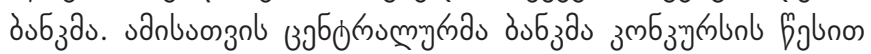

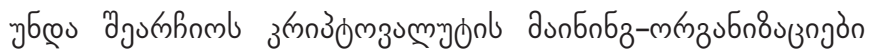

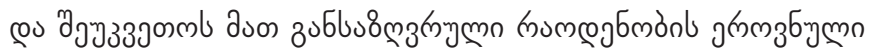

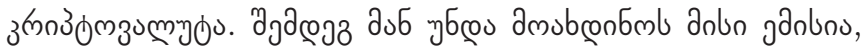

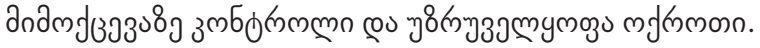

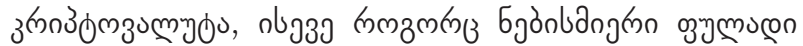
gromgymn, forndmuœ

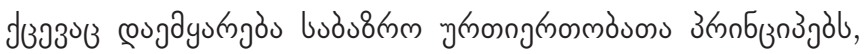

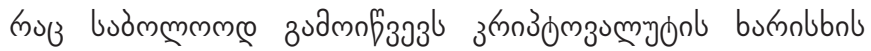

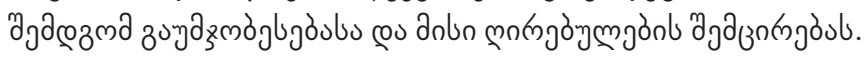

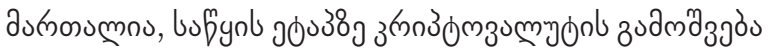

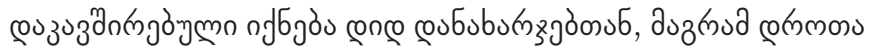

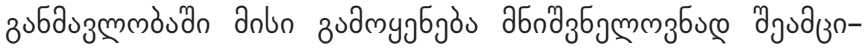

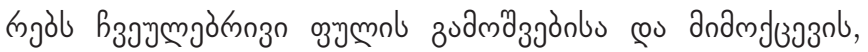

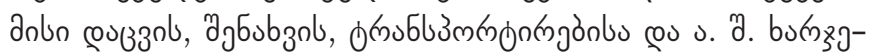

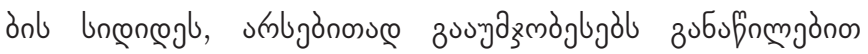

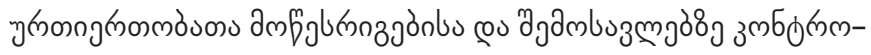

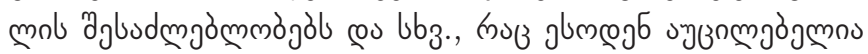

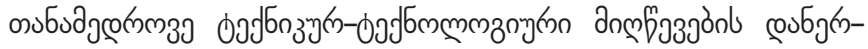

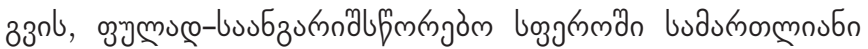

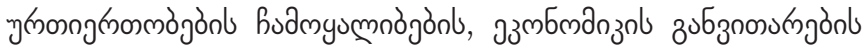

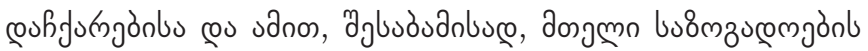

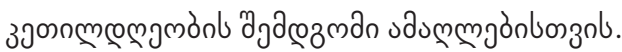




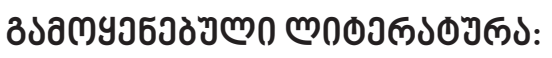

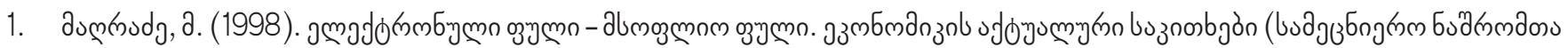

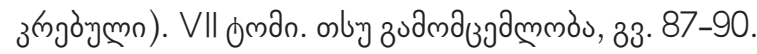

2. Букия Г. Б.Сичинава Д. Ш. и др. (1976).Общегосударственные автоматизированные системы безналичного расчета. Сообщение АН ГССР, том 83, №3, с. 117-123.

3. https://bit.ly/2MoDyOh

4. https://bit.ly/2LUviJl

5. https://bit.ly/2LS7zts

6. https://bit.ly/2KrMuAA

7. https://bit.ly/2AIGLXt

8. https://bit.ly/2Ko51Sh

9. https://bit.ly/2vAvFhm

10. https://bit.ly/2AL5omk

11. https://bit.ly/2AETM4h

12. https://bit.ly/2LUvOr3

13. https://bit.ly/2McdUfg

14. https://bit.ly/2ncfmn9

15. https://bit.ly/2tvTKWw

16. https://bit.ly/2Avsjlo

17. https://bit.ly/2OB9wIA

18. https://bit.ly/2n4pMFm

19. https://bit.ly/2u2PxcR

20. https://bit.ly/2LOGEyl

21. https://bit.ly/2AB9VHO

22. https://bit.ly/2ofPzOx

23. https://bit.ly/2MaJzOx

24. https://bit.ly/2n4Hfxm

25. https://bit.ly/2LZr2HY 


\section{PRECONDITIONS AND PROBLEMS OF TRANSITION TO ELECTRONIC MONEY}

\section{DEMUR SICHINAVA}

https://doi.org/10.35945/gb.2018.06.026

Professor,

Ivane Javakhishvili Tbilisi State University, Georgia

demur.sitchinava@tsu.ge

\section{MURTAZ MAGRADZE}

Professor, emeritus,

Ivane Javakhishvili Tbilisi State University, Georgia

murtaz.maghradze@tsu.ge

\section{KEYWORDS: ELECTRONC MONEY, CRYPTO CURRENCY, ELECTRONICWALET,}

HE MNING OF CRYPTO CURRENCY G GORGIA, PRLCONDIIONSTO TRANSIION TOLLCTRONIC MONEY

PROSPECTS OF CRYPTO CURRENCYDEVELOPMENT

\section{SUMMARY}

The article is dedicated to the actual problems of creation, intervention and introduction of electronic money crypto-currency and blockchain technology at the modern stage. Rather narrow description is given to essence, the history of invention and the types of crypto currency (bit coin, ethereum, etc.,), advantages and defects of their use, the electronic wallet and its types, situation of the electronic money introduc- tion in Georgia, the global crypto currency spread and the tendencies and prospective of its possible recognition, assessed is significance of the electronic money in manufacturing, based on the modern technical-technological achievements, money-reporting field, maximally just relations creation and by this - further growth of the economic development and accordingly the permanent enhancement of the living standards of the populations. 